\title{
Neural basis for social cognitive impairment in schizophrenia
}

\author{
Bases neurais do comprometimento da cognição social na esquizofrenia \\ Hironobu Fujiwara', Cali Bartholomeusz²
}

\section{SOCIAL COGNITION AND ITS NEURAL BASIS}

Social cognition is defined as "the mental operations underlying social interactions, which include the human ability to perceive the intentions and dispositions of others"1. This ability would be required in situations in which plural individual humans as well as primates are present, therefore, having good or superior social cognitive abilities would be advantageous for an individual's survival from an evolutionary perspective.

In recent years, insight into the neural basis of this ability has rapidly been growing with the use of functional and structural neuroimaging techniques and neuropsychological measures. Such evidence suggests that several different brain regions across the frontal, temporal, and parietal regions and limbic system, would play a role in social cognitive processing, with the construction of neural networks among each region. Among such widely ranged putative neural underpinnings of social cognition, the orbitofrontal cortex (OFC), amygdala (Amy), medial prefrontal cortex (MPFC), anterior cingulate cortex (ACC), insular cortex (IS), inferior parietal cortex, temporo-parietal junction (TPJ), superior temporal sulcus (STS) and fusiform gyrus (FG) have been consistently reported. For example, FG, STS, and Amy (which plays a critical role in recognizing emotions) would underlie emotional face processing. With respect to Theory of mind (ToM), the ability to infer the other's mental status, the TPJ, MPFC, ACC, and STS are the representative regions that have been repeatedly demonstrated as the neural underpinnings. In Empathic ability, ACC, Amy, OFC and IS are considered to be critical for this processing.

Taken together, the neural basis of the various different facets of social cognition overlap to some extent, however, these different facets of social cognition are considered to be processed by different neural networks among plural brain regions, and such variety of the network would make these complex cognitive processing, including complex social judgments, possible.

\section{SOCIAL COGNITIVE IMPAIRMENT IN SCHIZOPHRENIA}

A deficit in social functioning is one of the most disabling clinical features of schizophrenia (SCZ) and a significant factor in the resulting social isolation experienced by many patients. Several cognitive deficits, for example, memory, attention and working memory, would have an influence on the patient's social functioning, however, one factor leading to social dysfunction is miscommunication with others, which is, at least partially, the result of impaired social cognition². In daily clinical psychiatric practice, it is sometimes necessary to provide patients with rehabilitation therapy, such as social skills training, in addition to anti-psychotic medication therapy, which might imply the presence of social cognitive impairment in the disorder.

1 Department of Neuropsychiatry, Graduate School of Medicine, Kyoto University.

2 Melbourne Neuropsychiatry Centre, The University of Melbourne.

Endereço para correspondência: Hironobu Fujiwara

Department of Neuropsychiatry, Graduate School of Medicine, Kyoto University, 54 Kawara-cho, Shogoin, Sakyo-ku, Kyoto 606-8507, Japan

E-mail: fwiz3668@kuhp.kyoto-u.ac.jp 
Meanwhile, cumulative evidence of neuroimaging studies suggest that the neural basis of SCZ involves several brain regions, for example, MPFC, Amy, MPFC, ACC and temporal regions. These regions, as the neural basis of the disorder, remarkably overlap with those of social cognitive functions. This highlights the need to investigate social cognitive disabilities in SCZ and their associations with neuroimaging findings, which would lead to further understanding of the pathophysiology of schizophrenia. Below, neuroimaging studies are presented and discussed in terms of the neural underpinnings of different social cognitive domains.

\section{EMOTIONAL FACIAL RECOGNITION}

Although speech is the most straightforward tool for communications with others, it is also critical for social interactions to recognize non-verbal massages including eye gaze, gestures and facial expressions. Emotional (especially basic emotions including fear, happy, anger, sad, disgust, surprise) facial recognition is one of the most widely researched social cognitive processes. The neural system implicated in facial emotion recognition has been proposed to include the Amy, the FG and the STS ${ }^{3}$. In particular, Amy is suggested to be closely involved in the recognition of negative facial emotions, and this theory has been supported by both lesion studies and imaging studies. Human lesion studies have consistently found impaired recognition of facial emotion following bilateral Amy damage. This impaired recognition is often disproportionately prominent for recognizing the emotion fear. Moreover, recent functional (PET, SPECT and FMRI) imaging studies also supports the consensus that Amy and its related network is the neural underpinning of the processing of emotion recognition. Such deficits are repeatedly reported in SCZ, however, it is still possible that social cognitive impairments might be related to other general cognitive deficits, for example, dysexecutive function or memory dysfunction (although it is not always restricted to emotional facial recognition). In other words, it is very important to adequately control for confounding factors in the experimental design, in order to investigate whether such deficits are specific to the domain of social cognition.

\section{ToM}

To engage in successful social interaction, one must recognize that others have independent experiences and intentions, and perhaps even ulterior motives. The ability to represent another person's psychological perspective is referred to as $\mathrm{ToM}^{4}$ or mentalizing ${ }^{5}$. ToM allows us to predict the behavior of others. Various ToM tasks have been studied, including story and cartoon comprehension in social situations.
Several brain regions have consistently been suggested as the neural basis of ToM, included the TPJ, STS, the temporal poles, and the MPFC (for convenience, including ACC) ${ }^{6}$.ToM deficits have been reported early in the course of pervasive developmental disorders (PDD), and such impairment has also been suggested in SCZ. However, it is still obscure whether the deficits are unique or whether they are related to the symptomotology of the disorder, like affective flattening or impairment in other cognitive domains. Thus, further investigation would be needed to clarify the issue with strictly controlled conditions.

\section{EMPATHY}

The term "empathy" refers to the process of inferring and sharing the emotional experiences of others, in addition to representing what others are thinking (i.e. ToM). In this sense, empathy refers to affective facets of the ToM ability for example, imagining joy and sadness in others' minds, rather than ToM alone (i.e. only thinking about other's intentions without an emotional component). From this point of view, the neural bases of empathy and ToM would be hypothesized to be, at least partially, independent. Indeed, several functional imaging studies provide evidence that supports this hypothesis.

In an fMRI study that used a cartoon task (in which each story represented either ToM or empathy), brain activations during the ToM condition were relatively greater in the OFC, middle frontal and superior temporal regions than during the empathy condition. Conversely, greater activations in the anterior/posterior cingulate and Amy were observed during the empathy condition compared to the ToM condition ${ }^{7}$. As well, Hynes et al. ${ }^{8}$ applied several written sentences, in which affective and cognitive components of perspective taking (PT) are represented, as a paradigm to investigate the differences between the neural bases of these two facets of PT. As a result, differential subregions in OFC were suggested to be specifically responsible for each cognitive and emotional PT. Thus, although the overlap of the activations is present in different facets of empathy, differential processes should still be considered.

In respect to empathic disability in $\mathrm{SCZ}$, such deficits have been repeatedly suggested with self-rating questionnaires, for example the Interpersonal Reactivity Index $x^{9,10}$.

\section{NEUROIMAGING AND SOCIAL COGNITION IN SCHIZOPHRENIA}

The number of imaging studies showing neural underpinnings of social cognitive impairments in SCZ is getting larger in recent years. Particularly, lines of evidence in functional ima- 
ging studies suggest that social cognitive impairments are present in SCZ and their neural basis is widely spread across different brain regions, such as the Amy, IFG, IS and MPFC.

Several structural imaging studies have also provided knowledge of the relationship between social cognitive impairments and brain abnormalities in SCZ. Namiki et al."11, by using an MRI manual tracing method, suggested that a deficit of emotional facial recognition (particularly "fear" face recognition) was associated with volume reductions in Amy. Yamada et al. ${ }^{12}$, investigated the relationship between brain changes (assessed by voxel-based morphometry: VBM) and social cognitive disability of SCZ by using PAT (Perception of Affect Tasks) ${ }^{13}$, which are designed to assess the ability to infer others' emotions in situations which are closely related to actual daily life. As a result, the association of MPFC volume reduction with lower task performance was found in $\mathrm{SCZ}^{12}$. Additionally, using the same paradigm, Fujiwara et al. ${ }^{14}$ also demonstrated that lower PAT scores were associated with ACC volume reductions, as well as abnormal sulcal variation in SCZ. This indicates that ToM deficits could be both stateand trait- related because brain sulcal variation is generally stable after birth, while ACC volume change often occurs before as well as after the onset of SCZ.

In summary, the neural basis of social cognitive impairment in SCZ is gradually gaining some clarification as mentioned above, however, it is still unknown whether such deficits are related to other characteristics of SCZ pathology including basic cognitive dysfunction and symptomatology of the disorder. Furthermore, it is also still unclear whether social cognitive dysfunction is state-related or trait-related or both. In this sense, a small number of fMRI studies attempted to address these issues with longitudinal designs. Similarly, studies involving individuals at high-risk for psychosis have demonstrated differences in brain activity before and after the psychotic episode ${ }^{15}$, as well as differences between highrisk individuals who later developed psychosis and those who did not ${ }^{16}$. Further studies in these areas are needed to clarify above-mentioned questions in the future.

\section{REFERENCES}

1. Brothers $L$. The social brain: a project for integrating primate behavior and neurophysiology in a new domain. Concepts Neurosci. 1990;:27-51.

2. Lee KH, Farrow FD, Spence SA, Woodruff PWR. Social cognition, brain networks and schizophrenia. Psychol Med. 2004:43:391-400.

3. Adolphs R. Neural systems for recognizing emotion. Curr Opin Neurobiol. 2002;22:169-77.

4. Premack D, Woodruff G. Chimpanzee problem solving: a test for comprehension. Science. 1978;202:532-5

5. Frith U, Frith CD. Development and neurophysiology of mentalizing. Philos Trans R SoC London B. 2003;358:459-73.

6. Amodio DM, Frith CD. Meeting of minds: the medial frontal cortex and social cognition. Nat Rev Neurosci. 2006;7:268-77.

7. Völlm BA, Taylor AN, Richardson P, Corcoran R, Stirling J, McKie S, et al. Neuronal correlates of theory of mind and empathy: a functional magnetic resonance imaging study in a nonverbal task. Neuroimage. 2006;29:90-8.

8. Hynes CA, Baird AA, Grafton ST. Differential role of the orbital frontal lobe in emotional versus cognitive perspective-taking. Neuropsychologia. 2006;44:374-83.

9. Montag C, Heinz A, Kunz D, Gallinat J. Self-reported empathic abilities in schizophrenia. Schizophr Res. 2007;92:85-9.

10. Fujiwara H, Shimizu M, Hirao K, Miyata J, Namiki C, Sawamoto N, et al. Female specific anterior cingulate abnormality and its association with empathic disability in schizophrenia. Prog Neuropsychopharmacol Biol Psychiatry. 2008;32:1728-34.

11. Namiki C, Hirao K, Yamada M, Hanakawa T, Fukuyama H, Hayashi T, et al. Impaired facial emotion recognition and reduced amygdalar volume in schizophrenia. Psychiatry Res Neuroimaging. 2007;156:23-32.

12. Yamada M, Hirao K, Namiki C, Hanakawa T, Fukuyama H, Hayashi T, et al. Social cognition and frontal lobe pathology in schizophrenia: a voxel-based morphometric study. Neuroimage. 2007;35:292-8

13. Rau JC. Perception of verbal and nonverbal affective stimuli in complex partial seizure disorder [abstract]. Dissertation Abstracts Int B. 1993;54:506B.

14. Fujiwara H, Hirao K, Namiki C, Yamada M, Shimizu M, Fukuyama H, et al. Anterior cingulate pathology and social cognition in schizophrenia: a study of gray matter, white matter and sulcal morphometry. Neuroimage. 2007;36:1236-45.

15. Lee KH, Brown WH, Egleston PN, Green RD, Farrow TF, Hunter MD, et al. A functional magnetic resonance imaging study of social cognition in schizophrenia during an acute episode and after recovery. Am J Psychiatry. 2006;163:1926-33.

16. Marjoram D, Job DE, Whalley HC, Gountouna VE, McIntosh AM, Simonotto E, et al. A visual joke fMRl investigation into Theory of Mind and enhanced risk of schizophrenia. Neuroimage. 2006;31:1850-8. 